\title{
El estado de Bloqueo Difuso Generalizado. Un obstáculo interaccional a los procesos de aprendizaje en la organización
}

\section{The state of Generalized Diffuse Blocking. An interactional obstacle to organizational learning processes}

\section{Montenegro, Gustavo; Schroeder, Ivana Karina ${ }^{1}$}

Resumen. Los avances contemporáneos en el campo de la cognición humana y las perspectivas de la complejidad posibilitan pensar el fenómeno de la interacción social en la organización y en otras instituciones de la vida social considerando la amplia red de aspectos socioemocionales, comunicacionales, interaccionales y contextuales que operan generando situaciones de malestar, conflicto y violencia. En este trabajo presentamos algunos elementos sistematizados en el marco de la investigación en procesos de cambio y aprendizaje organizacional que dan cuenta de un patrón de bloqueo interaccional subyacente en la cotidianeidad institucional que ocasiona con frecuencia perjuicios intangibles a las posibilidades de satisfacción, pertenencia y salud personal y colectiva. Sostenemos que existe una relación de reforzamiento mutuo entre los comportamientos defensivos de los sujetos y la consolidación de reglas de interacción regresivas que operan en la organización naturalizando configuraciones anímicas que afectan los vínculos y las relaciones de igual manera que el logro de metas y objetivos.

Palabras clave: aprendizaje organizacional-cognición - sistemas complejos - violencia subyacente

\footnotetext{
${ }^{1}$ Montenegro, Gustavo. Licenciado en Psicología. UNC. Doctorando en Instituto de Investigación y Formación en Administración Pública (IIFAP) UNC. Profesor investigador de la Facultad de Psicología UNC. Profesor investigador del Instituto de Ciencias de la Administración (ICDA) UCC. Córdoba. Argentina. gustavo.montenegro@unc.edu.ar - ORCID: https://orcid.org/0000-0002-2684-9550

Schroeder, Ivana Karina. Licenciada en Psicología UNC. Especialista en Gestión de Organizaciones sin fines de Lucro. UCC. Profesora investigadora de la Facultad de Filosofía y Humanidades y del Instituto de Ciencias de la Administración (ICDA) UCC. Córdoba. Argentina.0216373@ucc.edu.ar - ORCID: https://orcid.org/00000002-7304-902X
}

Debates actuales en Psicología y Sociedad. ISSN en línea: 2591-524X

Año II, Nº 2, 2019. Pág. 30-52.

DOI: 10.22529/daps.2019.2(2)03 / Recibido: 3-8-2018 / Aprobado: 4-2-2019.

Artículo publicado bajo Licencia Creative Commons Atribución-NoComercial-SinDerivar.

(C) Universidad Católica de Córdoba. 


\begin{abstract}
Contemporary advances in the field of human cognition and the perspectives of complexity make it possible to think about the phenomenon of social interaction in the organization and in other institutions of social life considering the wide network of socioemotional, communicational, interactional and contextual aspects that operate generating situations of discomfort, conflict and violence. In this paper, we present some systematized elements within the framework of research in processes of change and organizational learning that account for an underlying interactional blocking pattern in the institutional daily life that frequently causes intangible damages to the possibilities of satisfaction, belonging and personal and collective health. We argue that there is a relationship of mutual reinforcement between the defensive behavior of the subjects and the consolidation of regressive interaction rules that operate in the organization naturalizing mood configurations that affect the links and relationships in the same way as the achievement of goals and objectives.
\end{abstract}

Keywords: organizational learning- cognition - complex systems - underlying violence 


\section{Introducción}

En el marco de lo que puede caracterizarse como una economía del conocimiento, la teoría organizacional viene asistiendo en las últimas décadas a cambios muy profundos asociados a los desafíos que impone la complejidad dinámica del contexto actual, en el que el valor del conocimiento como factor de desarrollo económico y social resulta ampliamente reconocido (Bueno Campos, 2004). Pensar la organización como un sistema viviente, una entidad cognitiva con capacidad de innovación, de aprendizaje y creación de saber viene desplazando a la visión maquinista propia del modelo de la organización tradicional burocrática.

En esa dirección, numerosas perspectivas contemporáneas como la Gestión del Conocimiento y el Aprendizaje Organizacional problematizan de manera diversa aspectos que hacen a la interacción cognitiva en la organización, en relación con las capacidades de aprendizaje, la creación de conocimiento y la innovación organizacional frente al abordaje de los problemas cotidianos emergentes. Entre ellos, se ha puesto particular foco a los procesos comunicacionales y los espacios de reflexividad colectiva, a la creatividad y los modelos de pensamiento, a la dinámica socioafectiva, a los procesos de decisión y resolución de problemas

Para la psicología de la organización la consideración de los procesos mentales y el estudio de la influencia del contexto organizacional sobre la conducta de las personas y los grupos ha resultado relevante desde sus inicios. Al respecto, los aportes de la psicología cognitiva contemporánea han puesto en relieve los fenómenos por los cuales los sujetos reciben, interpretan, almacenan, evalúan y utilizan la información en sus actividades de trabajo (Schein, 1997; Munduate Jaca, 1997).

Desde este trasfondo de supuestos, la investigación que llevamos adelante desde un enfoque cualitativo y una metodología situada en prácticas concretas de cambio asistido en pequeñas y medianas organizaciones de la ciudad de Córdoba, pretende responder a ¿por qué y de qué manera se producen -o no se producen- los fenómenos de aprendizaje organizacional?, ¿qué relación puede reconocerse entre los fenómenos de aprendizaje organizacional y los aspectos que hacen a la interacción cognitiva entre los actores?, y ¿cómo pueden caracterizarse el conjunto de estos elementos en términos de propiedades y dimensiones? En ese sentido, la investigación asume un interés particular por la problemática 
de la interacción cognitiva entendiendo que la capacidad de aprender requiere identificar los problemas y transformarlos en innovaciones del diseño organizacional que permitan superarlos y prevenirlos en el futuro.

Desde un enfoque teórico asentado en principios epistemológicos de la perspectiva de sistemas complejos y aportes del pragmatismo epistemológico y la cognición distribuida, el estudio persigue la inteligibilización de los procesos de afrontamiento de problemas, de los elementos de interacción cognitiva que median en su eficacia y de las condiciones organizacionales que intervienen en estos fenómenos. Un supuesto central que orienta a la investigación es que la calidad con la que se presente la dinámica de interacción cognitiva es decir, la calidad con la que se den los procesos comunicaciones, decisionales, socio anímicos y representacionales que median en cualquier intento de coordinación y entendimiento - presenta una incidencia en la capacidad de afrontamiento de los problemas (o quiebres) emergentes en la organización, y en su potencial transformación en aprendizajes e innovaciones organizacionales específicas.

La exploración y el análisis de las circunstancias de interacción crítica en la organización, nos ha llevado a identificar estados distintos de funcionamiento del sistema cognitivo organizacional en su conjunto, asociados a patrones de interacción cognitiva entre los actores y en la organización. En este trabajo presentamos avances y resultados en torno a uno de esos estados que hemos denominado Bloqueo Difuso Generalizado. En los casos estudiados, este estado del sistema se presenta en circunstancias en las que frente al quiebre observamos una relación de reforzamiento mutuo entre los comportamientos defensivos de los sujetos y la consolidación de reglas de interacción regresivas que van progresivamente naturalizando configuraciones anímicas que afectan los vínculos y las relaciones de igual manera que el logro de metas y objetivos.

Sostenemos que en tanto se naturalizan estas reglas y situaciones de tensión y conflicto vincular cotidiano, los actores institucionales son objeto de violencia naturalizada y recíproca que arraiga en fenómenos de bloqueo y clausura individual, grupal e institucional. Esta violencia de carácter simbólico y emergente de la dinámica interaccional institucional no es la clásica violencia institucional que emana necesariamente de las reglas de dominación hegemónica (tal cual fuera caracterizada por Bourdieu); sino que deviene más bien de debilidades o déficits de actualización del diseño de afrontamiento de los quiebres cotidianos. En todo caso, puede atribuírsele a la red de autoridad de conducción institucional ciertos 
márgenes de debilidad de agencia en el diseño de la estructura socio-institucional, lo que ocasiona con frecuencia perjuicios intangibles a las posibilidades de satisfacción, pertenencia y salud personal y colectiva.

\section{Perspectiva teórica}

La investigación se enmarca en un enfoque teórico multirreferencial que articula los aportes de diversos enfoques: a) el aprendizaje organizacional, b) la ciencia cognitiva contemporánea, en particular la perspectiva de la cognición distribuida, y c) la teoría de sistemas complejos.

a) Aprendizaje organizacional:

Comprender cómo aprenden las organizaciones viene siendo foco de atención e investigación en el campo del análisis organizacional y de la administración en las últimas décadas. El aprendizaje organizacional, y con ello, el proceso de creación y adquisición de conocimiento en una organización, no es un proceso natural o espontáneo, sino más bien un proceso que requiere de condiciones organizacionales que lo promuevan (Castañeda-Zapata, 2015). Numerosos autores consideran el aprendizaje organizacional como fenómeno estratégico en la dirección de organizaciones, sin embargo, coexisten interpretaciones múltiples respecto a los elementos, factores o variables que lo posibilitan. En la literatura académica $-\mathrm{y}$ precisamente más en ensayos teóricos que en estudios empíricos- el aprendizaje organizacional es asociado a la gestión del conocimiento (Porras Velásquez, 2015; Angulo Rincón, 2017); a una cultura de innovación para adecuarse a un entorno cambiante y así actualizarse y evolucionar (Sahui Maldonado y Patrón-Cortés, 2015; Gómez Rodríguez, 2016;), a la cultura organizacional abierta al cambio (Rostro Hernández y Solís Hernández, 2016), a la formación del personal (Castañeda-Zapata, 2015), entre otros.

A partir de los trabajos iniciales de Argyris y Schon (1978) sobre las rutinas defensivas y los obstáculos al aprendizaje en la organización, y las alusiones de Simon (1991) a la metáfora cognitiva, algunos estudios han puesto en foco la necesidad de congruencia entre los actores y los elementos cognitivos de la organización, con principal atención a los procesos de resolución de problemas, a los sujetos solucionadores y sus interacciones. En ese sentido han cobrado relevancia los procesos de comunicación, diálogo y reglas de racionalidad comunicativa y ampliada para la reflexividad colectiva (Nonaka y Takeuchi, 1995; Zarifian 1996, Dejours, 1998; Johnson y Lundvall, 1994; Lundvall, 1999; Argyris, 
2001; Rojas, 1999), la puesta en juego de estrategias de pensamiento sistémico y complejo (Senge, 1990; Checkland, 1993; Gómez-Degraves, 2012; Vera y Ospina, 2016; Muñoz; 2017; Hernández-Castro, Garza, Martínez y Barbosa-Álvarez, 2016); los modelos mentales (Romero, Maramoro y Campos, 2013), los procesos de decisión y resolución de problemas (Gore, 2003, 2006; Ospina, 2014; Gómez Romero, Villarreal-Solís y Villarreal-Solís, 2015, Fassio y Rutty, 2017).

En este contexto de perspectivas se sostiene que los niveles de comprensión y aprendizajes genuinos que demanda la organización contemporánea, descansan en la calidad de puesta en juego de rutinas de diálogo reflexivo para la identificación y transformación de los quiebres organizativos aportando así, a la recodificación de la memoria organizacional y el rediseño organizativo permanente (Rojas, 1999, Flores 1997). Un quiebre significa cualquier interrupción en devenir natural y no examinado de la acción, una perturbación de la experiencia. Representa, en el "método de zona de innovación" de Rojas (1999), la identificación precisa de una oportunidad de innovación y aprendizaje. Para Flores (1997) la clave consiste en identificar los quiebres recurrentes e implementar acciones de diseño tendientes a transformarlos, superarlos y prevenirlos en el futuro.

En el marco de nuestra investigación, los quiebres, así como sus circunstancias de superación, son tangibilizados en base a la conceptualización operacional de las experiencias de brecha entre realidad y expectativa que -experimentadas por los actores de manera en gran parte tácita- subyacen a las circunstancias de tensión crítica en la vida organizacional cotidiana. De tal manera, la observación de los quiebres organizacionales, así como las circunstancias de evolución y superación de los mismos, si bien puede ser tangibilizada en términos de criterios e indicadores conceptualizables -e inclusive pasibles de medición-, no puede objetivarse si no en íntima relación con la indagación de la experiencia subjetiva de los actores involucrados.

\section{b) Cognición distribuida:}

En el campo de la Ciencia Cognitiva se ha dado un profuso desarrollo de líneas teóricas e investigativas que ponen en relieve el carácter extendido, situado y emergente de la cognición. La concepción de lo "mental" como un fenómeno que trasciende a los sujetos individuales y a la función cerebral, y de los sistemas cognitivos como entidades que se apoyan en factores extra-craneales, está presente explícitamente en los desarrollos de Bateson 
(1998), y posteriormente conforma el núcleo fundamental de las perspectivas de la cognición distribuida (Salomon, 2001; Cole yEngestrom, 2001; Hatchy Gardner, 2001; Brown, Ash, y otros, 2001), la cognición extensa (ClarkyChalmers, 1998; Clark, 1999), la filosofía de la mente (Dennet, 1995, 1996, 2000, Dawkins, 2000, Broncano, 2006, Vélez, 2007; Vega, 2005), la cognición “encarnada” (Varela, Thompson y Rosch, 1992; Ortuzar, 2005), y la cognición situada (BeguinyClot, 2005).

La cognición distribuida plantea que los procesos sociales deben ser tratados como cogniciones, y que el entorno y los recursos físicos, simbólicos y sociales que se hallan fuera de la persona, participan en la cognición no solo como fuentes y receptores de suministros y productos sino como vehículos de pensamiento (Perkins,2001). En tal sentido, la cognición está distribuida física, social y simbólicamente, las personas piensan y recuerdan con ayuda de toda clase de instrumentos físicos y por medio del intercambio con otros, compartiendo información, puntos de vista e ideas (Salomon, 2001).

\section{c) Sistemas complejos:}

Si bien existe consenso en afirmar que no existe aún algo que se asemeje a una teoría unificada de la complejidad (Reynoso, 2006; Maldonado y Gómez Cruz, 2010; Rodríguez Zoya y Aguirre, 2011; Elizalde Prada, 2013), las ciencias de la complejidad presentan en las últimas décadas un desarrollo e interés creciente. El estudio de los problemas de complejidad organizada se ha nutrido de variedad de ciencias, metodologías, lenguajes, enfoques, teorías y disciplinas como la cibernética y la cibernética de segundo orden de Foerster; la epistemología genética de Piaget; la teoría de la auto-organización de Ashby, la teoría general de los sistemas de Bertalanffy; la geometría fractal de Mandelbrot; la teoría de los autómatas celulares de Neumann; la termodinámica de los procesos irreversibles de Prigogine; la teoría de la autopoiesis de Maturana y Varela; la teoría de las catástrofes de Thom, entre otras (Rodríguez Zoyay Aguirre, 2011).

En el campo de las ciencias sociales y humanas, disciplinas como la historia, la antropología, la arqueología, los estudios culturales, la economía, la sociología, la epistemología, la filosofía y la administración vienen incorporando elementos provenientes de las ciencias de la complejidad. En ese sentido parece haber una apertura o acercamiento de estas ciencias - variando énfasis e intereses - con el estudio de fenómenos caracterizados por 
no-linealidad, emergencia, autoorganización, equilibrios dinámicos y comportamientos aperiódicos (Maldonado, 2009).

En esa diversidad, las que cabría denominar perspectivas constructivistas de sistemas complejos, cercanas a los fundamentos del pragmatismo epistemológico (García, 2006; Le Moigne, 1990a, 1990b, 1993; Rodríguez Zoya y Roggero, 2014), se nos presenta como recurso pertinente para la inteligibilización de los procesos de aprendizaje organizacional, en la medida que la heterogeneidad de aspectos y niveles de análisis en juego en estos fenómenos ameritan un abordaje sistemático y articulado del conjunto de componentes estructurales y dinámicos involucrados.

En correlación con ello, la estructura de los sistemas se concibe como conformada por elementos heterogéneos, que se articulan de manera no descomponible en un marco de diversos niveles y escalas de conjuntos componentes internos. Estos niveles y escalas, a la vez que se constituyen bajo principios de funcionamiento semiautónomo, desarrollan particulares propiedades emergentes y establecen entre sí distintos grados de jerarquía, autonomía e interconexión recíproca. Tal es así que el conjunto estructural es definido dinámicamente por las relaciones entre sus componentes más que por los componentes en sí, y tales relaciones son en diverso grado susceptibles a fenómenos de estabilidad, alternancia y reorganización.

La consideración de fundamentos de las perspectivas de sistemas complejos nos aportan conceptos sensibilizadores para el estudio de los fenómenos de aprendizaje organizacional, y la comprensión de los fenómenos de estabilidad y cambio involucra la consideración de procesos de autoorganización, de principios de causalidad recíproca y no lineal entre los componentes en juego, así como la atención a fenómenos de amplificación de fluctuaciones, transiciones de fase y creación de nuevas estructuras en condiciones alejadas del equilibrio (García, 2006; Sotolongo Codina y Delgado Díaz, 2006; Maldonado, 2007, 2011; Prigogine, 2012).

\section{Planteo metodológico}

La investigación, de enfoque cualitativo, se plantea bajo la modalidad de diseño de estudio de casos contemporáneo (Martínez-Carazo, 2006; Neyman y Quaranta, 2007; Fassio, 2018) y apela, de manera complementaria, a fundamentos y procedimientos de la teoría fundamentada (Strauss y Corbin, 2002), de la ciencia acción (Schon, 1992; Rojas, 1999; 
Argyris, 2001) y de la perspectiva constructivista de sistemas complejos (García, 2006; Le Moigne, 1990a; Rodríguez Zoyay Aguirre, 2011).

La estrategia metodológica se focaliza en el mapeo y seguimiento de procesos de afrontamiento de problemas (quiebres) y la identificación de condiciones intervinientes en los fenómenos de aprendizaje organizacional ${ }^{2}$, y se desarrolla en base a un detenido análisis de prácticas de asistencia técnica en procesos de cambio y aprendizaje llevado adelante en 28 casos - constituidos por organizaciones por pequeñas y medianas organizaciones del sector privado, público y social- en la ciudad de Córdoba (Argentina), en el marco de un programa de investigación iniciado en 2010.

La indagación empírica se realiza mediante el diseño y aplicación de entrevistas semiestructuradas a protagonistas institucionales y referentes de asistencia técnica participantes de los procesos de cambio y aprendizaje organizacional planeado y asistido llevado adelante en los casos de estudio ${ }^{3}$. Estas entrevistas - individuales y grupales- tienen lugar en cuatro circunstancias distintas en el trascurso del proceso de cambio y aprendizaje organizacional: etapa diagnóstica, etapa de avance e implementación, circunstancias de impedimento o dificultad en el proceso y, situaciones de logro. Asimismo, tienen lugar en marcos de exploración distintos: entre investigador y técnico/s, entre investigador y protagonistas institucionales; entre técnicos y protagonistas; entre investigador, técnicos y protagonistas en conjunto. El diseño de entrevistas involucra tres tipos de preguntas: preguntas focalizantes, destinadas a la detección de situaciones singularmente significativas desde el punto de vista del entrevistado; preguntas exploratorias, que persiguen la caracterización de eventos, acciones o situaciones circunstancialmente significativas; y

\footnotetext{
${ }^{2}$ Cabe aclarar que las posibilidades de observabilidad y tangibilización de los elementos nucleares del estudio, como lo son los fenómenos de aprendizaje organizacional, los elementos interaccionales y cognitivos que constituyen el afrontamiento de situaciones problemáticas y el conjunto de elementos subjetivos y objetivos que operan como condiciones de tales procesos, representan un desafío metodológico particular. Estos fenómenos, por un lado, disponen de una débil manifestación tangible, lo cual dificulta su operacionalización, y, por otro lado, tal como se plantea desde diversos campos del conocimiento y la investigación, se presentan en gran parte de manera tácita y subyacente respecto a la conciencia y al lenguaje ordinario de los propios involucrados. Otro tanto ocurre con los quiebres, que naturalmente tienden a encubrirse y a operar de manera subyacente a las situaciones de tensión emergentes en la vida organizacional. En este sentido, la modalidad de entrevista ocupa un lugar relevante en el estudio.

${ }^{3}$ Desde las estudios en torno a la gestión de cambio organizacional, se han referido aspectos comunes del cambio planeado: es intencional, es positivo, refleja las circunstancias, se centra en los valores de la organización, se orienta a la acción y se enfoca en el aprendizaje (Romero, Maramoros y Campos, 2013).En el marco de este trabajo asumen un papel particular los profesionales responsables del proceso de asistencia técnica como agentes de cambio (externos), y como tales representan, junto con los protagonistas institucionales, una fuente principal de datos e información relevante a los fines del estudio.
} 
preguntas aperturantes que procuran la identificación de componentes inferenciales tácitos involucrados en la percepción, la acción, o la decisión del entrevistado.

Así planteada, la entrevista más que conformarse en las descripciones y estructuraciones narrativas que el entrevistado presenta de manera primaria, prevé un conjunto de procedimientos para la exploración activa de elementos de trasfondo intervinientes en los comportamientos e interacciones referidas por el entrevistado. Como parte de ello, y en un marco de acuerdos previamente establecidos, el entrevistador aporta al entrevistado una serie de procedimientos de orden meta cognitivo que intervienen de manera ampliatoria en las configuraciones perceptivas del entrevistado respecto a las situaciones y condiciones relevantes de los procesos observados.

El análisis de datos y el desarrollo conceptual se realiza particularmente mediante instancias de codificación, muestreo teórico y comparación constante propios de la teoría fundamentada. Basados también en fundamentos de la modelización de sistemas complejos, resultan relevantes aspectos particulares que hacen a la noción de explicación y causalidad por cuanto en una explicación causal la relación entre las situaciones empíricas es inferida a partir de la relación lógica dentro de la teoría (Garcia, 2006, p. 79) ${ }^{4}$.Complementariamente con ello, se apoya en sistemas de matrices de datos (Samaja, 2008) en tanto se brinda como recurso pertinente para la organización, definición y representación articulada de niveles, dimensiones, subdimensiones y estados relevantes.

En cuanto a previsiones de fiabilidad y validez, el desarrollo de la investigación ha contemplado la utilización de distintas fuentes de información complementarias en el proceso de recogida de evidencia, siguiendo el principio de triangulación. Se ha realizado triangulación de fuentes de datos incluyendo entrevistas individuales y grupales con participación cruzada de protagonistas institucionales y responsables de asistencia técnica y revisión documental de registros internos, informes de caso y protocolos de demanda. En atención al mantenimiento de la cadena de evidencia, los elementos de referencia empírica han sido registrados y clasificados en soporte informático Atlas ti.

${ }^{4}$ Crítico de la postura baconiana de la ciencia, según la cual se llega a las relaciones causales por vía inductiva, a través de una generalización de "regularidades" observables en la experiencia, García sostiene que en una explicación causal la relación entre las situaciones empíricas es inferida a partir de la relación lógica dentro de la teoría (García, 2006, p. 79). Para el autor, este planteo, según el cual las relaciones causales se establecen en el nivel teórico (aunque puedan ser "sugeridas" por la experiencia) implica reconocer que las relaciones entre observables (o entre hechos) no surgen de la simple "evidencia" empírica (p. 46). 


\section{Resultados}

Hemos referido que la exploración y el análisis de las circunstancias de interacción crítica en los casos en estudio, nos ha llevado en el proceso de investigación a diferenciar dos estados de funcionamiento diferente que el sistema organizacional puede asumir en su conjunto frente al quiebre. Ambos estados aparecen como circunstancias simétricamente contrapuestas en tanto representan dos destinos alternativos posibles de las situaciones de quiebre en la organización.

A nivel fenoménico, estos estados se plantean como un conjunto de manifestaciones perceptibles en torno a la efectividad, la calidad de relaciones y el bienestar de los actores en la organización, pero a nivel subyacente, como una conjunción de patrones de funcionamiento que actúan de manera correlacionada a nivel micro, meso y macro del sistema cognitivo organizacional. En consideración de la perspectiva de sistemas complejos que sustenta el estudio, la noción de "patrón" involucra reconocer en los fenómenos observados propiedades de autoorganización y emergencia. Esto supone afirmar que los distintos atributos mantienen entre sí relaciones de causalidad recíproca y también que las transiciones entre los distintos patrones se producen en base a principios de causalidad no lineal.

En términos dinámicos, el quiebre representa para el sistema una perturbación e implica una fluctuación. En estado estable, las fluctuaciones pueden resultar insignificantes, no relevantes, en cuyo caso son contenidas por el sistema. Pero en situaciones alejadas del equilibrio puede suceder lo contrario, en vez del retorno a un estado inicial, se advierte que las fluctuaciones se amplifican, y esta amplificación lleva a una situación nueva. Según Prigogine (2012), en condiciones alejadas del equilibrio, el sistema es capaz de percibir diferencias en el mundo exterior y de reaccionar con grandes efectos a pequeñas fluctuaciones.

En este sentido, y desde la perspectiva del aprendizaje organizacional, las circunstancias relevantes que tomamos, no surgen de la contraposición entre presencia o ausencia de quiebres, sino de la contraposición entre dos destinos - o respuestas posiblesante el quiebre. Dado que los quiebres son inerradicables de la vida organizacional, la pregunta se define en torno a la capacidad del conjunto social de transformar quiebres en aprendizajes organizacionales eficaces, así como por las condiciones y elementos operantes para que ello suceda - o no. 
Tal como hemos anticipado, en este trabajo presentamos avances y resultados en torno a uno de esos estados que hemos denominado Bloqueo Difuso Generalizado (BDG), en tanto se nos presenta como dificultad para el logro de aprendizajes organizacionales eficaces.

\section{El Estado de Bloqueo Difuso Generalizado}

El BDG se nos presenta como una circunstancia naturalizada en la que los quiebres y dificultades en el plano operacional se encuentran agudizadas por quiebres y dificultades en el plano comunicacional, vincular y estructural. En otras palabras, en la situación de BGD no sólo hay dificultades para resolver la tarea sino también en las condiciones de la relación, lo cual genera profundas dificultades para poder conversar productivamente sobre los problemas de tarea. Se trata de una circunstancia conformada en torno a un conjunto de inquietudes irresueltas en los actores que se manifiestan como irrupciones en el bienestar y la satisfacción, asociadas de manera diversa a los resultados o condiciones de la tarea, la calidad de relación, las reglas de funcionamiento, o las representaciones interpersonales mutuas.

El supuesto de base es que los quiebres que no son transformados oportunamente en aprendizaje organizacional e innovaciones eficaces, se transforman en tensiones difusas frecuentemente progresivas- que afectan la calidad de la tarea, las interacciones, los vínculos y las disposiciones personales. Dicho de otra forma, las dificultades persistentes en la tarea, las relaciones y el bienestar de las personas en una organización, tienen bases en quiebres subyacentes que no encuentran posibilidad de ser transformados en innovaciones eficaces del diseño organizacional. El sistema dinámico deviene inestable a causa de perturbaciones o estrés, que pasado cierto umbral crítico es impulsado ya sea a un nuevo orden o a la desintegración (Reynoso, 2006). Si bien el cambio podría ser proporcional a la perturbación, en ocasiones un cambio imperceptible en una constante producirá un cambio cualitativo en el comportamiento del sistema. Cualquier mínima perturbación lleva a la evolución dinámica del sistema hacia una de ellas, pudiendo combinar la estabilidad y el cambio en estadios sucesivos (García Azconobieta, 2005).

La observación y análisis de las circunstancias de BGD a la luz de los procedimientos de la teoría fundamentada y los sistemas de matrices de datos (Samaja, 2008), nos permiten identificar un conjunto de componentes que, bajo principios de funcionamiento semiautónomo, desarrollan propiedades emergentes particulares y establecen entre sí distintos grados de interconexión recíproca. Esto ha sido referido como el principio de organización 
sistémica (García, 2006). Es así que se registran, entre otros aspectos, dificultades, insatisfacciones y aumento de incidentes y tensiones en la interacción operativa, así como desajustes y divergencias en el diseño operacional puesto en juego, y desacomodamiento o zonas difusas en la memoria operacional. A nivel comunicacional, se advierte cierta predominancia de juegos conversacionales de confrontación y encubrimiento o "privados", con incidencia de trasfondos interpretativos centrados en juicos personales y/o grupales que van naturalizando narrativas subgrupales disfuncionales e inorgánicas. Se naturalizan canales informales de resolución comunicacional y operativa que aumentan las criticidades metacomunicacionales asociadas a los modos de decir o actuar. En el plano decisorio, se registran determinadas debilidades en el proceso de tratamiento de los quiebres, así como la consolidación de unilateralidad cognitiva y decisional en torno a esos procesos. Hay un debilitamiento del trasfondo compartido y del contexto de plausibilidad orgánico, se naturaliza la existencia de barreras defensivas asociadas a configuraciones anímicas cierre y desconfianza. Tales fenómenos dan cuenta de un patrón cognitivo de clausura como racionalidad predominante.

Estos fenómenos inherentes al BDG, se encuentran asociados a lo que Argyris y Schon -en el marco de su teoría de la acción- señalaron como Modelo I de comportamiento, caracterizado por comportamientos defensivos creados por los individuos y reforzados por la cultura de la organización. Estas acciones, a la vez que son apeladas para eludir situaciones incómodas, problemáticas y peligrosas, impiden a los actores identificar y reducir las causas de tales situaciones. Se trata de un proceso circular de reforzamiento mutuo entre las acciones defensivas de los individuos y la cultura de la organización. Estas rutinas defensivas y conductas de anti-aprendizaje sobreprotegen a los individuos y las organizaciones y los inhiben de aprender nuevas acciones. Las rutinas defensivas ocurren constantemente y son independientes de la personalidad de los sujetos individuales, se ejecutan de forma automática y en muchas ocasiones sin atención consciente (Argyris, 1999).

Como parte de la conformación del BGD, se naturalizan también circunstancias cotidianas de simulación, sabotaje, desprestigio, actitudes amenazantes, desconsideración de expectativas y esfuerzos recíprocos en el marco de las cuales los actores son sumidos en procesos anímicos de resignación, angustia o resentimiento que muestran considerables afecciones al bienestar y proyección personal y a la salud personal y colectiva. 
El registro de entrevistas, nos ha permitido identificar múltiples referencias al BDG aludido por los actores participantes en los casos estudiados. En palabras expresadas por ellos: “...no nos entendemos, pero tampoco hay voluntad de mejorar nada (Entrevista 28, caso E), “...ellos están convencidos de que hacen todo bien, y todos los días hay algún problema, si no son los turnos son los papeles de archivo, pero ni siquiera se puede hablar..." (Entrevista 45, caso M), “...no, ya ni lo planteo, para qué si nadie escucha, hago la mía y listo" (Entrevista 83, caso T). En expresiones como estas se hacen manifiestas de manera convergente dificultades para el entendimiento, la coordinación de acciones y la eficacia conjunta. Así como acciones que remiten a violencia simbólica manifestada en disposiciones anímicas de resentimiento y angustia, presencia de barreras defensivas interpersonales, sensaciones de desencanto e intolerancia mutua y percepciones del otro como amenaza; actitudes de culpabilización recíproca, manipulación unilateral, indiferencia a las expectativas mutuas y disputa sostenida por determinados bienes materiales o simbólicos en juego.

En palabras de los referentes de asistencia técnica también se hace alusión al estado de bloqueo: “... hay un malestar generalizado que afecta la tarea cotidiana, pero además a la relación y a las disposiciones individuales, a lo que incluso parecen haberse acostumbrado...” (Entrevista AT16), “...están debilitadas las vías formales de intercambio, y el circuito decisional inorgánico se ha vuelto natural a pesar de que la gente manifiesta las disrupciones que esto ocasiona" (Entrevista AT22). En muchas ocasiones el conjunto de los aspectos mencionados es acompañado por una naturalización del malestar, e inevitablemente y con frecuencia de manera subyacente y no consciente, por determinadas insatisfacciones de los actores respecto a expectativas específicas (quiebres).

En tanto circunstancia naturalizada, en el marco de BGD se van instituyendo progresivamente, a modo de "pautas culturales", ciertas reglas que van moldeando las disposiciones personales y el comportamiento en torno a qué se hace con lo que satisface y no satisface, cómo se comunica, cómo se conversa y quién conversa con quién, quién y cómo decide y con qué niveles de involucramiento, cómo se configuran y piensan los problemas y qué se hace con las tensiones, el malestar y el conflicto. Estos conjuntos dinámicos de reglas aun siendo de difícil visibilización y explicitación- estructuran los procesos de tratamiento de problemas, de tensiones y conflictos emergentes, y configuran las modalidades de decisión, de operación y gestión cotidiana dificultando e incluso impidiendo el logro de aprendizajes organizacionales eficaces. 


\section{Conclusiones}

Como bien han señalado algunos autores, las dificultades que operan para que el aprendizaje organizacional no sea posible, o se vea limitado, en generalhan sido tratadas como un tema secundario, pero estudiarlas resulta de vital importancia dado que, si no se identifican y se superan, el aprendizaje en las organizaciones será deficiente y estará plagado de errores desde su generación hasta su generalización.

En ese sentido, hemos pretendido en este trabajo dar cuenta de avances y resultados del proceso de investigación sobre procesos de aprendizaje organizacional, asentado en prácticas concretas de asistencia técnica en pequeñas y medianas organizaciones de la ciudad de Córdoba. En los casos estudiados, hemos observado circunstancias en las que frente al quiebre emerge una relación de reforzamiento mutuo entre los comportamientos defensivos de los sujetos y la consolidación de reglas de interacción regresivas que de manera progresiva naturalizan configuraciones anímicas que afectan los vínculos y las relaciones de igual manera que el logro de metas y objetivos. A la luz del enfoque del aprendizaje organizacional, de los aportes de la ciencia cognitiva y de perspectiva de sistemas complejos que sustentan teóricamente el estudio, hemos denominado a esta circunstancia estado de Bloqueo Difuso Generalizado (BDG).

El BGD constituye un patrón de actividad del sistema en su respuesta ante los quiebres emergentes, que se manifiesta de manera sensible para nosotros a través de las distintas modalidades de actuación e interacción cognitiva de los actores.

Nos parece relevante señalar que la estabilización y causalidad de las modalidades de comportamiento en los actores, no obedecen predominantemente a factores relativos a la cognición individual, sino que supone un conjunto de transiciones de estado de diversa escala en distintos componentes y niveles del sistema de cognición organizacional. En otras palabras, las condiciones de unilateralidad, desconfianza y aislamiento, asociadas a situaciones de malestar y conflicto institucional -no siempre explícitas-, involucran pensar la organización como un sistema cognitivo con modalidades alternativas de funcionamiento (o racionalidades) que están muy lejos de ser únicamente voluntad de los sujetos individuales.

Puede observarse recurrentemente en el diagnóstico y la asistencia técnica organizacional cómo los actores institucionales son objeto de malestar interaccional, vincular, operativo y simbólico, en asociación con una cristalización de situaciones sostenidas y 
naturalizadas de tensión y conflicto interpersonal o inter grupal. En estas situaciones, la estabilización de barreras defensivas y representaciones mutuas de desconfianza se van apropiando de las reglas implícitas de interacción haciendo que la salud y la potencia de las personas, de las relaciones entre ellas y del proyecto organizacional se degradan a niveles considerables, pero a veces invisibilizados.

Es posible interpretar estas problemáticas con el hecho de que las instituciones de la modernidad clásica, - familias, organizaciones del trabajo, instituciones educativas, proyectos sociales- han visto vulneradas sus claridades de funcionamiento, sin que necesariamente se hayan desarrollado y consolidado en ellas nuevos dispositivos y reglas de accionar potente y saludable que reclama la dinámica organizacional actual. En relación con ello cabe observar los desafíos de conciencia reflexiva y de agencialidad sobre las estructuras organizativas en los distintos espacios y niveles de las instituciones de la vida social contemporánea. Resulta esperable que, en tanto avanza la capacidad organizacional para la identificación de quiebres emergentes y la puesta en juego sustentable de procesos de aprendizaje organizacional, disminuya la proporción de quiebres que devienen en patrones de bloqueo y clausura.

Finalmente, corresponde aludir a algunas limitaciones del estudio relacionadas con la proveniencia de los registros empíricos, en tanto refieren todos ellos a casos que, si bien acontecen en organizaciones diversas, comparten un mismo tipo de orientación estratégica respecto a los procesos de cambio y aprendizaje organizacional en transcurso. Dada la perspectiva de investigación adoptada, el relevamiento de antecedentes investigativos respecto a diversas unidades conceptuales involucradas, lejos está de ser agotado. De hecho, en el marco de la investigación en curso, seguimos profundizando en este punto.

Así también advertir que - a la luz de los principios epistemológicos y ontológicos de sistemas complejos que orientan la investigación-el conjunto de proposiciones presentadas en este trabajo guarda ciertos márgenes de provisionalidad y que las relaciones causales expuestas, si bien son sugeridas por la experiencia empírica, se establecen en un nivel teórico. Todo ello es postulado en el marco de un proceso de aproximaciones sucesivas hacia la inteligibilización e intento de modelización de la organización como sistema complejo de cognición distribuida. 


\section{Referencias Bibliográficas}

Angulo Rincón, R. (2017). Gestión del conocimiento y aprendizaje organizacional: una visión integral. Informes Psicológicos, 17(1), 53-70. https://doi:10.18566/infpsic.v17n1a03

Argyris, C. y Schon, D. (1978). Organizacional Learning. A theory of action perspective. Massachussets: Addison

Argyris, C. (1999). Conocimiento para la acción. Buenos Aires: Granica.

Argyris, C. (2001). Sobre el Aprendizaje Organizacional. México: Oxford University Press.

Bateson, G. (1998). Pasos hacia una ecología de la mente. Buenos Aires: Lohlé - Lumen.

Beguin, P. yClot, Y. (2005). L'actionsituéedans le développement de l'activité. Activités, 1 (2), 36-49.

Broncano, F. (2006). Sujeto y subjetividad en la mente extensa. Revista de Filosofía, 31(2), 109-133.

Brown, A., Ash, D. y otros. (2001). El conocimiento especializado distribuido en el aula. En G. Salomon (Comp.). Cogniciones distribuidas. Consideraciones psicológicas y educativas (pp. 242-290). Buenos Aires: Amorrortu.

Bueno Campos, E. (2004). Fundamentos epistemológicos de dirección del conocimiento organizativo: desarrollo, medición y gestión de intangibles. Economía Industrial (357), 13-26. Recuperado de:

http://mincotur.gob.es/Publicaciones/Publicacionesperiodicas/EconomiaIndustrial/Revista EconomiaIndustrial/357/03_EduardoBueno_357.pdf

Castañeda-Zapata, D. (2015). Condiciones para el aprendizaje organizacional. Estudios Gerenciales, 31, 62-67. https://doi:10.1016/j.estger.2014.09.003

Checkland, P. (1993). Pensamiento de sistemas, prácticas de sistemas. México: Grupo Noriega.

Clarck, A. y Chalmers, D. (1998). The extended mind. Analysis, 58 (1), 7-19.

Clark, A. (1999). Estar ahí. Cerebro, cuerpo y mundo en la nueva ciencia cognitiva. Barcelona: Paidós. 
Cole, M. y Engestrom Y. (2001). Enfoque histórico-cultural de la cognición distribuida. En G. Salomon (Comp.). Cogniciones distribuidas. Consideraciones psicológicas y educativas (pp. 23-74). Buenos Aires: Amorrortu.

Dawkins, R. (2000). El gen egoísta. Barcelona: Salvat.

Dejours, C. (1998). El factor humano, Buenos Aires: PIETTE/CONICET.

Dennett, D. C. (1995). La conciencia explicada: una teoría interdisciplinar. Buenos Aires: Paidós

Dennett, D. C. (1996). Contenido y conciencia. Barcelona: Gedisa.

Dennett, D. C. (2000). Tipos de mentes. Hacia una comprensión de la conciencia. Madrid: Debate.

Elizal de Prada, Ó. A. (2013). Aproximación a las ciencias de la complejidad. Revista de la Universidad de la Salle, (61), 45-66. Recuperado de: https://revistas.lasalle.edu.co/index.php/ls/article/view/2438/2155

Fassio, A. (2018). Reflexiones acerca de la metodología cualitativa para el estudio de las organizaciones. CienciasAdministrativas, 6, 73-84. https://doi:10.24215/23143738e028

Fassio, A. y Rutty, M. G. (2017). Hacia la identificación de dimensiones relevantes relacionadas con el aprendizaje organizacional. Trilogía Ciencia Tecnología Sociedad, 9 (16), 85- 99.

Flores, F. (1997). Inventando la empresa del siglo XXI. Santiago de Chile: Granica.

García, R. (2006). Sistemas complejos. Conceptos, método y fundamentación epistemológica de la investigación interdisciplinaria. Barcelona: Gedisa.

Gómez-Degraves A. (2012) Statistical-methodological proposal to measure organizational intelligence, based on the fifth discipline by Peter Senge. Negotium, 22 (9), 53-83. Recuperado de: http://revistanegotium.org.ve/pdf/22/art3.pdf

Gómez Rodríguez, M. (2016). El aprendizaje organizacional y su influencia en la innovación dentro de la organización. Revista Ingeniería, Matemáticas y Ciencias de la Información, 2(4). Recuperado de: http://ojs.urepublicana.edu.co/index.php/ingenieria/article/view/260 
Gómez-Romero, J.; Villarreal-Solís, M. y Villarreal-Solís, F. (2015). Las incapacidades de aprendizaje organizacional y su relación con los estilos de aprendizaje organizacional en la Mipyme. Contaduría y Administración, 60, 686-72. https://doi:10.1016/j.cya.2013.11.001

Gore, E. (2003). Conocimiento Colectivo. Buenos Aires: Granica.

Gore, E. (2006). Aprendizaje y Organización. Barcelona: Granica

Hatch, T. y Gardner, H. (2001). El descubrimiento de la cognición en el aula: una concepción más amplia de la inteligencia humana. En G. Salomon (Comp.), Cogniciones distribuidas. Consideraciones psicológicas y educativas (pp. 214-241). Buenos Aires: Amorrortu.

Hernández-Castro, N.; Garza, Z.; Martínez, E. y Barboza-Álvarez, V. (2016). Análisis de las dimensiones del aprendizaje Organizacional en empresas de la región Centro de Coahuila. Revista Internacional Administración \& Finanzas, 9 (7), 19-31. Recuperado de: http://www.theibfr2.com/RePEc/ibf/riafin/riaf-v9n7-2016/RIAF-V9N7-2016-2.pdf

Johnson, B. y Lundvall, B. A. (1994). Sistemas nacionales de innovación y aprendizaje institucional. Comercio exterior, 44 Recuperado de: http://revistas.bancomext.gob.mx/rce/magazines/362/4/RCE4.pdf

Le Moigne, J. L. (1990a). La modélisation des systemes complexes. Paris, Dunod: Collection Afcet Systeme.

Le Moigne, J. L. (1990b). Systemique et complexité: études d'épistémologies y stémique. Revue Internationales de Systémique, 4 (2), 107-117.

Le Moigne, J. L. (1993). La "incoherencia epistemológica" de las ciencias de la gestión. RevuePrancaise de Gestion 96. Traducido con autorización del autor por Ricardo Romero, Profesor de la Universidad Nacional de Colombia.

Lundvall, B. A. (1999). La base del conocimiento y su producción. Ekonomiaz: Revista vasca de economía,45. Recuperado de https://dialnet.unirioja.es/servlet/articulo?codigo=265836

Maldonado, C. (2007). Complejidad: ciencia, pensamiento y aplicaciones. Bogotá: Editorial, Universidad Externado de Colombia. 
Maldonado, C. E. (2009). Complejidad de los sistemas sociales: un reto para las ciencias sociales. Cinta de Moebio, 36, 146-157. http://doi.org/10.4067/S0717$\underline{554 X 2009000300001}$

Maldonado, C. (2011). Termodinámica y complejidad. Una introducción para las ciencias sociales. Bogotá: Desde Abajo.

Maldonado, C. E. y Gómez Cruz, N. A. (2010). Documentos de investigación. Facultad de Administración No. 76. El mundo de las ciencias de la complejidad Un estado del arte. Universidad del Rosario. Recuperado de: http://repository.urosario.edu.co/bitstream/handle/10336/3301/Fasc\%C3\%ADculo76.pdf? $\underline{\text { sequence }=1 \& \text { is Allowed }=y}$

Martínez-Carazo, P.C. (2006). El método de estudio de caso. Estrategia metodológica de la investigación científica. Pensamiento y Gestión, 20, 165-193.

Munduate, J. (1997). Psicología social de la organización. Las personas organizando. Madrid: Pirámide.

Muñoz, L. (2017). Propuesta para el desarrollo del pensamiento sistémico en los programas de formación de posgrado del convenio USTA-ICONTEC. Signos, 9 (2), 121-148. https://doi: 10.15332/s2145-1389.2017.0002.08

Neiman, G. y Quaranta, G. (2007). Los estudios de caso en la investigación sociológica. En I. Vasilachis de Gialdino (Ed.), Estrategias de investigación cualitativa (pp. 213-238). Buenos Aires: Gedisa.

Nonaka, I. y Takeuchi, H. (1995). The knowledge-creating company: How Japanese companies create the dynamics of innovation. New York, USA: Oxford UniversityPress.

Ortuzar, M.J. (2005). La Representación Mental en las Teorías "Encarnadas" Contemporáneas de la Cognición. (Tesis de maestría). Universidad de Chile. Recuperado de: http://www.repositorio.uchile.cl/handle/2250/108866

Ospina, Y. (2014). Aprendizaje Organizacional del Sujeto que Labora. Revista de Investigación en Ciencias Estratégicas, 1(1), 1-19. Recuperado de: http://revistas.upb.edu.co/index.php/RICE/article/view/2312/2062. 
Perkins, D. (2001). La persona-más: una visión distribuida del pensamiento y el aprendizaje. En G. Salomon (Comp.), Cogniciones distribuidas. Consideraciones psicológicas y educativas (pp. 126-152). Buenos Aires: Amorrortu.

Porras Velásquez, N. (2015). Gestión del conocimiento, aprendizaje y socialización organizacional: procesos fundamentales para la psicología del trabajo. Revista Poiésis. (29).https://doi:10.21501/issn.1692-0945

Prigogine, I. (2012). El nacimiento del tiempo. Buenos Aires: Tusquets Ed.

Reynoso, C. (2006). Complejidad y el caos: Una exploración antropológica. Buenos Aires: Editorial Sb, Colección Complejidad Humana.

Rodríguez Zoya, L. y Aguirre, J. (2011). Teorías de la complejidad y Ciencias Sociales. Nuevas estrategias epistemológicas y metodológicas. Nómadas. Critical Journal of Social and JuridicalSciences, 30 (2). Recuperado de: http://www.redalyc.org/articulo.oa?id=18120143010

Rodríguez Zoya., L. y Roggero, P. (2014). La modelización y simulación computacional como metodología de investigación social. Polis, 13(39). Recuperado de: https://scielo.conicyt.cl/pdf/polis/v13n39/art19.pdf

Rojas, E. (1999). El saber obrero y la innovación en la empresa. Montevideo: CINTERFOR/ OIT.

Romero, J., Maramoros, S. y Campos, C. (2013). Sobre el cambio organizacional. Una revisión bibliográfica. Revista INNOVAR, 23 (50), 35-52. Recuperado de: http://www.fce.unal.edu.co/media/files/innovar/v23n50/v23n50.pdf

Rostro Hernández, P. y Solís Hernández, O. (2016). Cultura y aprendizaje organizacional como base para el desarrollo regional. Revista Observatorio de la Economía Latinoamericana. Recuperado de: http://www.eumed.net/cursecon/ecolat/mx/2016/cultura.html

Sahui Maldonado, J. y Patrón-Cortés, R. (2015). Influencia del aprendizaje organizacional en el desarrollo de una actitud generadora de una cultura de innovación en las empresas. Revista Iberoamericana de Producción Académica y Gestión Educativa, 2(4). Recuperado de: http://www.pag.org.mx/index.php/PAG/article/view/308/351 
Salomon, G. (2001). No hay distribución sin la cognición de los individuos: un enfoque interactivo dinámico. En G. Salomon (Comp.), Cogniciones distribuidas. Consideraciones psicológicas y educativas (pp. 153-184). Buenos Aires: Amorrortu.

Samaja, J. (2008). Epistemología y metodología. Buenos Aires: Eudeba

Schein. E. (1997). Psicología de la Organización. México: Prentice Hall.

Schon, D. (1992). La formación de profesionales reflexivos. Hacia un nuevo diseño de la enseñanza y el aprendizaje en las profesiones. Madrid: Paidós Ibérica

Senge, P. (1990). La quinta disciplina. Buenos Aires: Granica

Simon, H. A. (1991): Bounded rationality and organizational learning. Organization Science, $2,(1), 125-134$.

Sotolongo Codina, P. L., y Delgado Díaz, C. J. (2006). La complejidad y el diálogo transdisciplinario de saberes. En La revolución contemporánea del saber y la complejidad social. Hacia unas ciencias sociales de nuevo tipo (pp. 65-77). Buenos Aires: CLACSO. Recuperado de: https://goo.gl/pzvhdt

Strauss A. y Corbin, J. (2002). Bases de la investigación cualitativa. Técnicas y procedimientos para desarrollar la teoría fundamentada. Bogotá: Editorial Universidad de Antioquia.

Varela, F, Thompson, E., y Rosch, E. (1992). De cuerpo presente. Barcelona: Gedisa.

Vega, J. (2005). Mentes hibridas: cognición, representaciones externas y artefactos epistémicos. Revista de Antropología Iberoamericana, (1). Recuperado de: https://dialnet.unirioja.es/ejemplar/125133

Vélez, J. C. (2007). El papel de los artefactos epistémicos en la nueva ciencia cognitiva. Cali: Universidad del Valle.

Vera, M. y Ospina, Y. (2016). Importancia del aprendizaje organizacional como mecanismo que favorece la consecución de los objetivos organizacionales. Colección Académica de Ciencias Sociales, 3, (1), 10-41. Recuperado de: https://revistas.upb.edu.co/index.php/cienciassociales/article/view/6190/5682 
El estado de Bloqueo Difuso Generalizado.

Un obstáculo interaccional a los procesos de aprendizaje en la organización

Zarifian, P. (1996). Cooperación, competencia y comunicación en el trabajo. En Travail et communication. París: PUF. 